\title{
Shot-noise anomalies in nondegenerate elastic diffusive conductors
}

\author{
G. Gomila \\ Research Center for BioElectronics and NanoBioScience, Departament d'Electronica, Universitat de Barcelona, C/ Marti i Franques, 1 , \\ E-08028 Barcelona, Spain \\ T. González \\ Departamento de Física Aplicada, Universidad de Salamanca, Plaza de la Merced s/n, E-37008 Salamanca, Spain \\ L. Reggiani \\ INFM-National Nanotechnology Laboratory, Dipartimento di Ingegneria dell'Innovazione, Università di Lecce, Via Arnesano s/n, \\ I-73100 Lecce, Italy
}

(Received 12 December 2001; revised manuscript received 21 March 2002; published 31 December 2002)

\begin{abstract}
We present a theoretical investigation of shot-noise properties in nondegenerate elastic diffusive conductors. Both Monte Carlo simulations and analytical approaches are used. Two interesting phenomena are found: (i) the display of enhanced shot noise for given energy dependences of the scattering time, and (ii) the recovery of full shot noise for asymptotic high applied bias. The first phenomenon is associated with the onset of negative differential conductivity in energy space that drives the system towards a dynamical electrical instability in excellent agreement with analytical predictions. The enhancement is found to be strongly amplified when the dimensionality in momentum space is lowered from three to two dimensions. The second phenomenon is due to the suppression of the effects of long-range Coulomb correlations that takes place when the transit time becomes the shortest time scale in the system, and is common to both elastic and inelastic nondegenerate diffusive conductors. These phenomena shed different light in the understanding of the anomalous behavior of shot noise in mesoscopic conductors, which is a signature of correlations among different current pulses.
\end{abstract}

DOI: 10.1103/PhysRevB.66.245423

PACS number(s): 73.23.- b, 73.50.Td, 72.70.+m, 05.40.-a

\section{INTRODUCTION}

The trend of scaling down the spatial dimensions of conducting samples is a demanding issue for realizing advanced electron devices and at the same time a fascinating realm for investigating new physical phenomena. Mesoscopic systems are presently a subject of intensive research and, being at intermediate sizes between atomic and micrometric structures, are in the position to satisfy this issue. In particular, a lot of attention is devoted to mesoscopic diffusive conductors, which are characterized by a sample length $L$, much smaller than the inelastic mean free path $l_{i n}$, and much longer than the elastic mean free path $l_{e l}$. These systems exhibit shot noise ${ }^{1}$ when the applied voltage $V$ is much higher than the thermal voltage $V_{T}=k_{B} T / q$, where $k_{B}$ is the Boltzmann constant, $T$ the bath temperature, and $q$ the unit charge. Shot noise can be conveniently studied in terms of the Fano factor $\gamma$ defined as $\gamma=S_{I}(0) /(2 q I)$, where $S_{I}(0)$ is the low-frequency current spectral density and $I$ the electric current. A value of $\gamma=1$ (full shot noise) implies the absence of any correlations among different current pulses, while $\gamma$ $\neq 1$ is a signature of the presence of correlations. In this latter case, both suppressed $(\gamma<1)$ and enhanced $(\gamma>1)$ shot noise are known to be possible.

In degenerate mesoscopic conductors at $T=0,{ }^{2,3}$ the correlations induced by the Pauli exclusion principle give rise to a universal Fano factor $\gamma=1 / 3$. By contrast, in nondegenerate mesoscopic conductors ${ }^{4-11}$ the Fano factor is in general a function of the applied bias and its value depends on the interplay between the long-range Coulomb interaction and the energy dependence of the elastic-scattering time. For the relevant case of a three-dimensional conductor (in momentum space) with an energy dependence of the scattering time of the form $\tau \propto \varepsilon^{\alpha}$, Monte Carlo (MC) simulations ${ }^{4-6}$ have reported a systematic analysis of the Fano factor in the range of values $-1.5<\alpha<1$. Results showed values of $\gamma$ close to $1 / 3$ for $\alpha$ between 0 and 1 and $V$ ranging between 40 and $120 V_{T}$. On the other hand, the values of $\gamma$ were found to increase systematically from $1 / 3$ towards 1 for values of $\alpha$ decreasing from 0 to -1.5 . The existing analytical theories $^{7-11}$ provide a reasonable interpretation of these results in the limited range of values $-1.5<\alpha<0.5$, being absent $^{10,11}$ or failing completely ${ }^{7-9}$ outside this range. ${ }^{6}$ In this context, two of the authors ${ }^{12}$ have recently pointed out the possibility that the above results only provide a partial description of the shot-noise properties of nondegenerate conductors. In particular, enhanced shot noise (i.e., $\gamma>1$ ) was predicted for given values of $\alpha$, and full shot noise (with $\gamma$ $=1$ ) was expected at asymptotically high bias for $\alpha$ $>-3 / 2$. We conclude that the scenario of shot noise in nondegenerate diffusive conductors, while phenomenological rich of interesting features, is still incomplete and waiting for a microscopic physical interpretation.

The aim of this work is precisely to address this issue. To this purpose, we have performed extensive MC simulations for a nondegenerate elastic diffusive conductor covering values of $\alpha$, applied bias, and dimensionality that are necessary for a unifying and complete analysis of the physical problem, and that enable a valuable test of the theoretical predictions ${ }^{12}$ to be carried out. The paper is organized as follows. Section 
II describes the physical model and the MC technique used in the calculations. Section III summarizes the theoretical predictions. Section IV presents the results of MC simulations proving the possibility for nondegenerate elastic diffusive conductors to display enhanced as well as full shot noise. The comparison between elastic and nonelastic transport regimes is also carried out to outline an interesting anomalous crossover from thermal to full shot noise exhibited by diffusive conductors. Section V draws the main conclusions of this work.

\section{PHYSICAL MODEL}

Following previous works, ${ }^{4-6}$ nondegenerate diffusive conductors are modeled as a simple structure consisting of a moderately doped semiconductor active region of length $L$ and constant cross section $A$ sandwiched between two heavily doped contacts that, by injecting carriers into the active region, act as ideal thermal reservoirs. ${ }^{13}$ According with the nondegenerate nature of the carrier injection and the thermal character of contacts, carriers are injected into the structure following a Maxwellian velocity distribution at the lattice temperature $T$ and a Poissonian time statistics, with injection rate $\Gamma=(1 / 2) n_{c} v_{t h}$, where $n_{c}$ is the contact density and $v_{t h}=\sqrt{2 k_{B} T / \pi m}$ the thermal velocity of injected carriers, with $m$ being the carriers effective mass. Once carriers are injected, their dynamics (under the action of scattering) is simulated in the active region of the structure by means of an ensemble MC self-consistently coupled with a Poisson solver (with constant voltage conditions at the boundaries) to account for Coulomb correlations. ${ }^{5}$ If not stated otherwise, we assume that the scattering processes taking place in the active region of the sample are elastic and isotropic and that the energy dependence of the scattering time is of the form $\tau(\varepsilon)=\tau_{0} \varepsilon^{\alpha}$. In principle, the exponent $\alpha$ can take any value, although only some particular values correspond to wellknown scattering mechanisms in a semiconductor model. As examples, $\alpha=-1 / 2$ corresponds to scattering with acoustic phonons by deformation potentials, $\alpha=0$ to neutral impurities, $\alpha=1 / 2$ to acoustic piezoelectric phonons, and $\alpha=3 / 2$ to short-range ionized impurity scattering. ${ }^{14}$ All these scattering mechanisms are important at low temperatures, which represent the typical conditions for an experimental test of theoretical predictions.

The structure is assumed to be sufficiently thick in transversal dimensions to allow for a one-dimensional electrostatic treatment. Accordingly, the Poisson equation is only solved in the direction of the current flow. In computer simulations one can consider any value of the momentum-space dimensionality $d$. Thus, even if the physically relevant case is $d=3$, we will analyze also the case $d=2$, since for this value of $d$ there exist analytical predictions based on the same assumptions on what concerns the coupling of the kinetic equation with the Poisson equation. ${ }^{7-12}$ As indicated in Ref. 8, this case corresponds to a hypothetical "flatland" whose physical realization would be a layered material with each layer containing a two-dimensional electron gas.

Due to their very high carrier concentration as compared to that of the active region, contacts are assumed to have no voltage drop inside them. The applied voltage between the contacts is then considered to be constant in time. As concerns noise calculations, this situation corresponds to current-mode operation. ${ }^{15}$

In our analysis of shot noise we are mostly interested in determining $S_{I}(0)$, which, in MC simulations, is obtained from the time integration of the autocorrelation function of current fluctuations $C_{I}(t)$. The calculation of $C_{I}(t)$ is performed from the time series $I(t)$ provided by the MC simulation. $I(t)$ is the instantaneous steady-state total current as measured in the outside circuit, which, for the assumed geometry, is also the same through each cross-sectional area of the device. For the semiclassical case considered here, the calculation of $I(t)$ is performed starting from the definition of the microscopic single-particle distribution,

$$
f(\mathbf{k}, \mathbf{r}, t)=\sum_{i=1}^{N(t)} \delta\left[\mathbf{k}-\mathbf{k}_{i}(t)\right] \delta\left[\mathbf{r}-\mathbf{r}_{i}(t)\right],
$$

where $N(t)$ is the instantaneous number of electrons inside the sample, which implies the conduction current density,

$$
\mathbf{j}_{c}(\mathbf{r}, t)=q \int \mathbf{v} f(\mathbf{k}, \mathbf{r}, t) d \mathbf{k}=q \sum_{i=1}^{N(t)} \mathbf{v}_{i}(t) \delta\left[\mathbf{r}-\mathbf{r}_{i}(t)\right],
$$

where $\mathbf{v}_{i}(t)$ is the instantaneous group-velocity determined through a band-structure model in $\mathbf{k}$ space. We notice that the above distribution function does contain fluctuations and has the following properties. Its ensemble average satisfies the Boltzmann equation ${ }^{16}$ while, in the linear approximation of small deviations from its average stationary value, it satisfies the Boltzmann-Langevin equation,

$$
\left(\frac{d}{d t}+S\right) f(\mathbf{k}, \mathbf{r}, t)=y(\mathbf{k}, \mathbf{r}, t),
$$

with $S$ the linearized scattering operator and $y(\mathbf{k}, \mathbf{r}, t)$ the fluctuating Langevin source related to $S .{ }^{16}$ From the solenoidal property of the total current density, and noticing that the displacement current is implicitly taken into account by constant-voltage conditions, it is ${ }^{15}$

$$
I(t)=\frac{q}{L} \sum_{i=1}^{N(t)} v_{i}(t)
$$

where $v_{i}(t)$ is the instantaneous carrier velocity along the field direction, known from the MC simulation. We remark that under steady-state conditions $I(t)$ is a stochastic quantity which enables the calculation of average values and correlation functions of its fluctuations with respect to average values. The basic sources of fluctuations in our system come from the instantaneous changes of carrier velocity due to scattering mechanisms and of carrier number inside the device due to injection-extraction processes. Thus velocity, number, and their cross-correlation contributions to the total current fluctuations are naturally accounted for together with their coupling with the fluctuating self-consistent field. We remark also that the ensemble MC simulation, by applying semiclassical dynamics to describe the motion of each particle between scattering events, is tantamount to a calculation 
of the microscopic distribution function $f(\mathbf{k}, \mathbf{r}, t)$ which, by a suitable discretization of phase space, could be collected during the simulation. Since two-particle interaction is here neglected (i.e., the corresponding Boltzmann equation is linear) and the collision rates are Markovian, the present ensemble MC simulation provides a numerical solution of the Boltzmann-Langevin equation (3).

Here we are primarily interested in $C_{I}(t)$. This quantity is directly calculated from the MC by the following procedure. After neglecting an initial transient, the values of $I(t)$ are recorded in a time grid of step size $\Delta t$ along the total simulation time $M \Delta t$, with $M$ integer. Then, by defining the time length in which the correlation function should be calculated as $m \Delta t$, with $m$ integer, $C_{I}(t)$ is obtained from the algorithm ${ }^{15}$

$$
\begin{aligned}
C_{I}(j \Delta t)= & \overline{I\left(t^{\prime}\right) I\left(t^{\prime}+j \Delta t\right)}-\bar{I}^{2} \\
= & \frac{1}{M-m} \sum_{i=1}^{M-m} I(i \Delta t) I[(i+j) \Delta t] \\
& -\left(\frac{1}{M} \sum_{i=1}^{M} I(i \Delta t)\right)^{2},
\end{aligned}
$$

where the bar denotes time average (ergodicity is implicitly assumed), and $j=0,1, \ldots, m ; M>m$. Typically, in our calculations $M>500 \times m, m>500$. The corresponding spectral density $S_{I}(f)$ is determined by Fourier transformation of the $C_{I}(t)$ so obtained.

For the calculations we have used the following parameters: $T=300 \mathrm{~K}$, effective mass $m=0.25 m_{0}$, with $m_{0}$ the free electron mass, $\epsilon=11.7 \epsilon_{0}$ (with $\epsilon_{0}$ the vacuum permittivity), $L=200 \mathrm{~nm}$ and $n_{c}=4 \times 10^{17} \mathrm{~cm}^{-3}$. For these values $L / L_{D_{c}}=30.9 \gg 1$. To test theoretical predictions we have performed simulations for systems with $d=2,3$, and the following values of $\alpha$ (and associated values of $\tau_{0}$ ) -2 (8.35 $\left.\times 10^{-16} \mathrm{~s} \mathrm{eV}^{2}\right), \quad-3 / 2 \quad\left(6.76 \times 10^{-16} \mathrm{~s} \mathrm{eV}^{3 / 2}\right), \quad-1 \quad(3.88$ $\left.\times 10^{-16} \mathrm{~s} \mathrm{eV}\right),-1 / 2\left(4.41 \times 10^{-16} \mathrm{~s} \mathrm{eV}^{1 / 2}\right)$, and $0(2.00$ $\times 10^{-15} \mathrm{~s}$ ). The values of $\tau_{0}$ are chosen in such a way to ensure diffusive transport through a sufficiently high number of scattering events (over $10^{2}$ ) in the active region. The Poisson equation is solved on a space mesh with 100 nodes. The number of simulated particles ranges between 100 and 2000 depending on $\alpha$ and bias conditions. Also depending on $\alpha$, the time step used to solve the Poisson equation ranges between 2 and $0.1 \mathrm{fs}$. The numerical uncertainty is confined within $10 \%$ in average and $20 \%$ at worst.

\section{THEORETICAL PREDICTIONS}

Before presenting the Monte Carlo results, we will briefly summarize and elaborate the theoretical predictions derived in Ref. 12 concerning the shot-noise properties of nondegenerate elastic diffusive conductors. In that work, by means of a formal treatment of the Boltzmann-Langevin-Poisson set of equations it was shown that the Fano factor for this class of systems can be exactly decomposed into the sum of three contributions as

$$
\gamma=\gamma_{i n}+\gamma_{\phi}+\gamma_{i n, \phi} .
$$

The first contribution, $\gamma_{i n}$, is related to the intrinsic current fluctuations in the presence of a static non-self-consistent electric potential. The second contribution, $\gamma_{\phi}$, is related to the current fluctuations induced by the fluctuations of the self-consistent potential. The third contribution, $\gamma_{i n, \phi}$, is related to the cross correlations between the previous two sources of fluctuations. Under far from equilibrium conditions (i.e., $V / V_{T}>3$ ), and for a nondegenerate conductor, the two following properties are satisfied: $\gamma_{i n}=1$ and $\gamma_{\phi} \geqslant 0$. As a consequence of these properties, theory predicts the following three regimes of shot noise: (i) enhanced shot noise when $\gamma_{i n, \phi}>-\gamma_{\phi}$, (ii) full shot noise when $\gamma_{\phi}=-\gamma_{i n, \phi}$, (iii) suppressed shot noise when $\gamma_{i n, \phi}<-\gamma_{\phi}$. Concerning enhanced shot noise, Eq. (6) and the properties $\gamma_{i n}=1, \gamma_{\phi} \geqslant 0$ imply that $\gamma_{i n, \phi}>0$ is a sufficient condition to obtain enhanced shot noise. This condition was found to be physically realized in the presence of a negative differential conductivity in energy space, i.e., $\sigma^{\prime}(\varepsilon)=d \sigma(\varepsilon) / d \varepsilon<0$, where the conductivity in energy space is given by ${ }^{7,12}$

$$
\sigma(\varepsilon)=\frac{1}{d} q^{2} \nu^{2}(\varepsilon) \nu(\varepsilon) \tau(\varepsilon)
$$

with $\nu(\varepsilon)=\Omega m\left(2 m \varepsilon / h^{2}\right)^{d / 2-1}$ the density of states in a $d$-dimensional momentum space, $\varepsilon$ the kinetic energy, $m$ the effective mass, $h$ the Planck constant, and $\Omega$ $=2 \pi^{d / 2} / \Gamma(d / 2)$ the surface area of the unit sphere in $d$ dimensions with $\Gamma(z)$ being the gamma function. Moreover, $v(\varepsilon)=\sqrt{2 \varepsilon / m}$ is the velocity and $\tau(\varepsilon)$ the elastic-scattering time. Therefore for $\tau(\varepsilon)=\tau_{0} \varepsilon^{\alpha}$ it is

$$
\sigma^{\prime}(\varepsilon)=C(\alpha+d / 2) \varepsilon^{\alpha+d / 2-1},
$$

where $C$ is a positive constant. We conclude that $\alpha<-d / 2$, by implying $\sigma^{\prime}(\varepsilon)<0$, represents a sufficient condition for the presence of enhanced shot noise. This constitutes the main prediction of the theory ${ }^{12}$ which is here under test.

Concerning full shot noise, Eq. (6) and the property $\gamma_{i n}$ $=1$ imply that $\gamma_{\phi}+\gamma_{i n, \phi}=0$ is a sufficient condition to obtain full shot noise since it corresponds to independent current pulses through the device. This condition can be realized by the two alternatives $\gamma_{\phi}=\gamma_{i n, \phi}=0$ or $\gamma_{\phi}=-\gamma_{i n, \phi} \neq 0$. The first alternative, $\gamma_{\phi}=\gamma_{i n, \phi}=0$, corresponds to washing out completely the effect of long-range Coulomb correlations. It can be realized under three means: (i) on a space scale, through the condition that $L<L_{D_{c}}$ where $L_{D_{c}}$ $=\sqrt{\epsilon k_{B} T / q^{2} n_{c}}$ is the contact Debye screening length calculated using the concentration of carriers at the contacts, with $\epsilon$ the static dielectric constant of the semiconductor and $n_{c}$ the contact concentration; (ii) on a time scale, through the condition $\tau_{T}<\tau_{d}$, where $\tau_{T}$ is the transit time and $\tau_{d}$ the dielectric relaxation time, when $L>L_{D_{c}}$; and (iii) on the energy dependence of the scattering time, through the condition for the energy exponent $\alpha=-d / 2$ (thus generalizing to any dimensionality the pioneer three-dimensional result of Nagaev $^{10}$ ). 
Condition (i) was tested by numerical simulations ${ }^{5}$ that showed full shot noise for $V / V_{T}>3$ at Debye lengths comparable with the sample length.

In condition (ii), being the sample length longer than the Debye screening length, there is room for Coulomb correlations to be effective. However, since the transit time depends on the bias, the onset of shot noise will start at voltages in general higher than those satisfying the condition $V / V_{T}>3$. The bias value at which shot noise will appear can be roughly estimated from the equality $\tau_{T}=L^{2} / \mu V_{\text {shot }}=\tau_{d}$ $=\epsilon / q \mu n_{c}$, with $\mu$ being an effective mobility. This gives $V_{\text {shot }}=q n_{c} L^{2} / \epsilon=\left(k_{B} T / q\right) L^{2} / L_{D_{c}}^{2}$, which, since $L>L_{D_{c}}$, will lead to $V_{\text {shot }} \gg V_{T}$. Therefore in this case we expect an anomalous transition between thermal and shot noise for biases $V_{T}<V<V_{\text {shot }}$. It is worth noting that the expression for $V_{\text {shot }}$ just derived coincides with the bias at which $L=L_{s}$, where $L_{s}=\sqrt{\epsilon V / q n_{c}}$ is the length used to characterize the space-charge limited conditions. ${ }^{8}$ This makes the condition for the appearance of full shot noise $\tau_{T}<\tau_{d}$ equivalent to the condition $L_{s}<L$, thus explaining why the existing theories, ${ }^{7-9,11}$ which apply for $L_{s}>L$, have failed in predicting the asymptotic presence of full shot noise. In any case, these theories are expected to remain valid for biases $V_{T}$ $<V<V_{\text {shot }}$, and their predictions can be taken to explain at least partially the anomalous crossover between thermal and shot noise where $\gamma$ takes values below unity.

In condition (iii), the crossover between thermal and full shot noise is monotonous and the Fano factor will not take values below one for any bias.

As regards the second alternative to obtain full shot noise, $\gamma_{\phi}=-\gamma_{i n, \phi} \neq 0$, we have not found physically feasible conditions to accomplish it. From the previous discussion, it follows that there are two main situations which should be tested by a direct microscopic simulation, namely, the possibility to observe enhanced shot noise for $\alpha<-d / 2$ at different dimensionalities, and the possibility to observe full shot noise for $L>L_{D}$ and $V>V_{\text {shot }}$.

\section{MONTE CARLO RESULTS}

In this section we report the results of MC simulations with the objective of providing a complete picture of the shot-noise properties of nondegenerate elastic diffusive conductors and of testing the theoretical predictions formulated in Sec. III. Initially we will illustrate the different conditions under which enhanced, suppressed, and full shot noise can be obtained in elastic diffusive conductors. Then, the crossover between thermal and shot noise will be detailed by comparing the noise power of an elastic diffusive conductor with that of an inelastic one.

\section{A. Enhanced, suppressed, and full shot noise}

Figure 1 reports the Fano factor as a function of the normalized voltage for a major set of simulations performed with different values of the exponent $\alpha$. Figure 1(a) refers to the three-dimensional (3D) case and Fig. 1(b) to the 2D case. The inset in each figure displays the $I-V$ characteristics in terms of the saturation current $I_{s}=q \Gamma A=(1 / 2) q n_{c} v_{t h} A$,

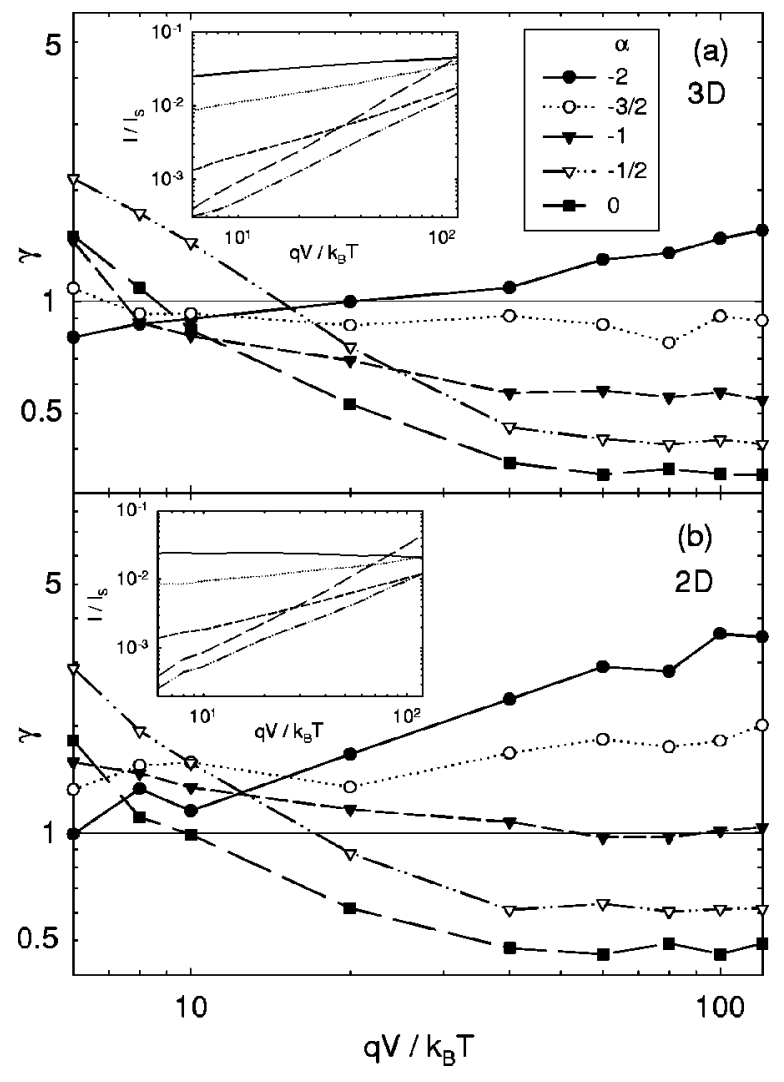

FIG. 1. Fano factor as a function of the applied voltage normalized to the thermal value for $\alpha=-2,-3 / 2,-1$, and 0 . Inset: $I-V$ characteristics with $I$ normalized to the saturation value $I_{s}$. (a) $3 \mathrm{D}$ system, (b) 2D system.

where $A$ is the cross-sectional area of the structure. For voltages below $10 V_{T}$ the conductor approaches thermal noise conditions, which explains a systematic trend of $\gamma$ to values greater than unity. By contrast, in the relevant region of voltages from 10 to $100 V_{T}$ the Fano factor is found to move from values smaller than 1 to values larger than 1 at decreasing values of $\alpha$. We note that for values of $\alpha=-2<-3 / 2$ in three dimensions $(\alpha=-3 / 2<-1$ in two dimensions) the sample displays enhanced shot noise at increasing voltages, in complete agreement with theoretical predictions for $d$ $=2$ and $d=3$. For values of $\alpha>-3 / 2$ in three dimensions (and $\alpha>-1$ in two dimensions) the system displays suppressed shot noise at increasing voltages, thus confirming the trend found in previous simulations. ${ }^{6}$ For $\alpha=-3 / 2$ in three dimensions (and $\alpha=-1$ in two dimensions) the system is found to display full shot noise within numerical uncertainty, as expected from theoretical predictions. Finally, by comparing the general trend of the Fano factor with the $I-V$ characteristics we find that in passing from suppressed to enhanced shot noise the conductor exhibits a substantial non-Ohmic behavior with a nearly saturation current regime at the largest negative $\alpha$ values.

To detail the evolution from suppressed to enhanced shot noise, we have reported in Fig. 2 the value of the Fano factor for the 3D and 2D cases at $V / V_{T}=60$ for all values of $\alpha$ considered here. For the purpose of comparison, the figure also reports the theoretical predictions of Refs. 7,10 and 11 


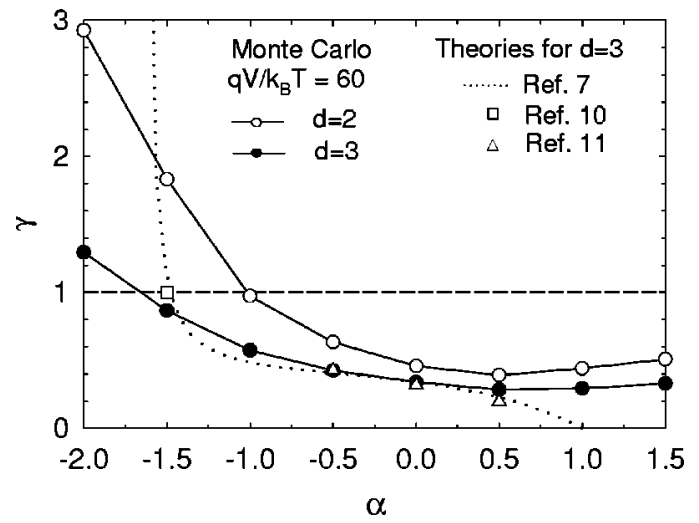

FIG. 2. Fano factor as a function of the energy exponent of the elastic-scattering time $\alpha$. Full circles and open circles refer to MC simulations performed at $V=60 V_{T}$ for $d=3$ and $d=2$, respectively. Dotted curve and other symbols refer to analytical theories carried out under space-charge limited conditions.

for $d=3$. The Fano factor is found to exhibit an asymmetric behavior with $\alpha$, showing a tendency to saturate, taking suppressed values near to $1 / d$, at positive values of $\alpha$, while increasing systematically towards enhanced values well above unity at the largest negative values of $\alpha$. The quantitative agreement between MC simulations and existing theories is found to be reasonably good for $-1.5<\alpha<0.5$. These theories are absent or fail completely outside this range of values. By contrast, starting from $\alpha \leqslant-d / 2$ the simulations evidence an enhanced shot-noise regime which fully validates the theoretical expectations. ${ }^{12}$

To investigate the mechanism responsible for the onset of shot-noise enhancement, Fig. 3 reports the spatial profiles of the relevant average quantities (concentration, electric field, velocity, and scattering time) for an applied voltage $V / V_{T}$ $=80$ and for different values of $\alpha$ in a 3D system. ${ }^{17}$ From the figure we identify the signature of a qualitative change in the different profiles for values of $\alpha$ below or about -1 . Here, we find the onset of two maxima (minima) in the velocity (concentration), more pronounced the lower the value of $\alpha$. This feature determines the presence of two regions inside the sample characterized by two transport regimes: a quasiballistic one in the region near to the contacts and a diffusive one around the center of the structure. Furthermore, a wide region of dynamical negative differential mobility shows up inside the sample, which in turns moves the structure towards a state of electrical instability, as is well known for the analogous case of Gunn diodes. ${ }^{18}$ These qualitative changes are accompanied by a systematic increase of the Fano factor, as can be seen in Figs. 1 and 2, what indicates the onset of a mechanism inducing positive correlations among the fluctuations. Therefore the increase of the Fano factor stems as a precursor of the fact that the system is evolving towards such an electrical instability. The appearance of a negative differential resistance in the $I-V$ characteristics (more pronounced in the 2D case), see insets of Fig. 1, further supports the present interpretation.

Figure 4 reports the spatial profiles of the same relevant quantities of Fig. 3 at different applied voltages for the 3D case with $\alpha=-2$. Here the onset of the bimodal profiles of

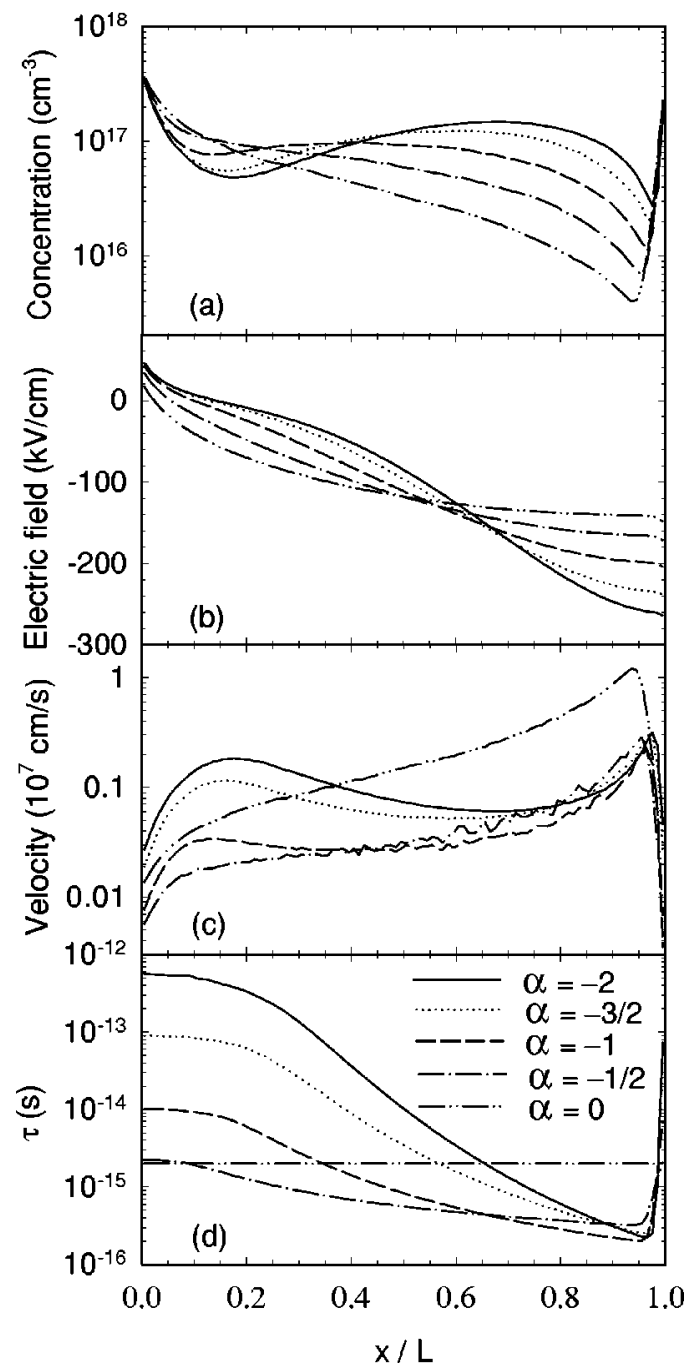

FIG. 3. Spatial profiles of (a) carrier concentration, (b) electric field, (c) average velocity, and (d) relaxation time along the active region of the sample as obtained by $\mathrm{MC}$ calculations for a $3 \mathrm{D}$ system, an applied bias of $V=80 V_{T}$, and several values of $\alpha$.

carrier concentration and velocity at increasing voltages is clearly confirmed, thus completing the analysis of the microscopic mechanism responsible of the shot-noise enhancement started with Fig. 3.

The existing MC simulations ${ }^{4-6}$ and analytical theories $^{7-11}$ have considered applied bias up to values high enough to reach the conduction regime known as spacecharge limited conditions and defined by the condition ${ }^{8} L$ $\gg L_{s} \gg L_{D_{c}}$, or equivalently $V_{T} \ll V \ll V_{\text {shot }}$. However, in Sec. III, starting from suppressed shot noise we have predicted the possible achievement of full shot noise for bias values satisfying the condition $V>V_{\text {shot }}$, which is in general beyond the space-charge limited regime. To test this prediction, we have performed MC simulations for a 3D system with scattering parameters $\alpha=3 / 2$ and $\tau_{0}=8.8 \times 10^{-15} \mathrm{~s} \mathrm{eV}^{-3 / 2}$, at applied voltages sufficiently high to satisfy $V>V_{\text {shot }}$. The results of such simulations are reported in Fig. 5, which shows the low-frequency current spectral density (continuous curve and full dots) and the current (multiplied by $2 q$, dotted curve) as 


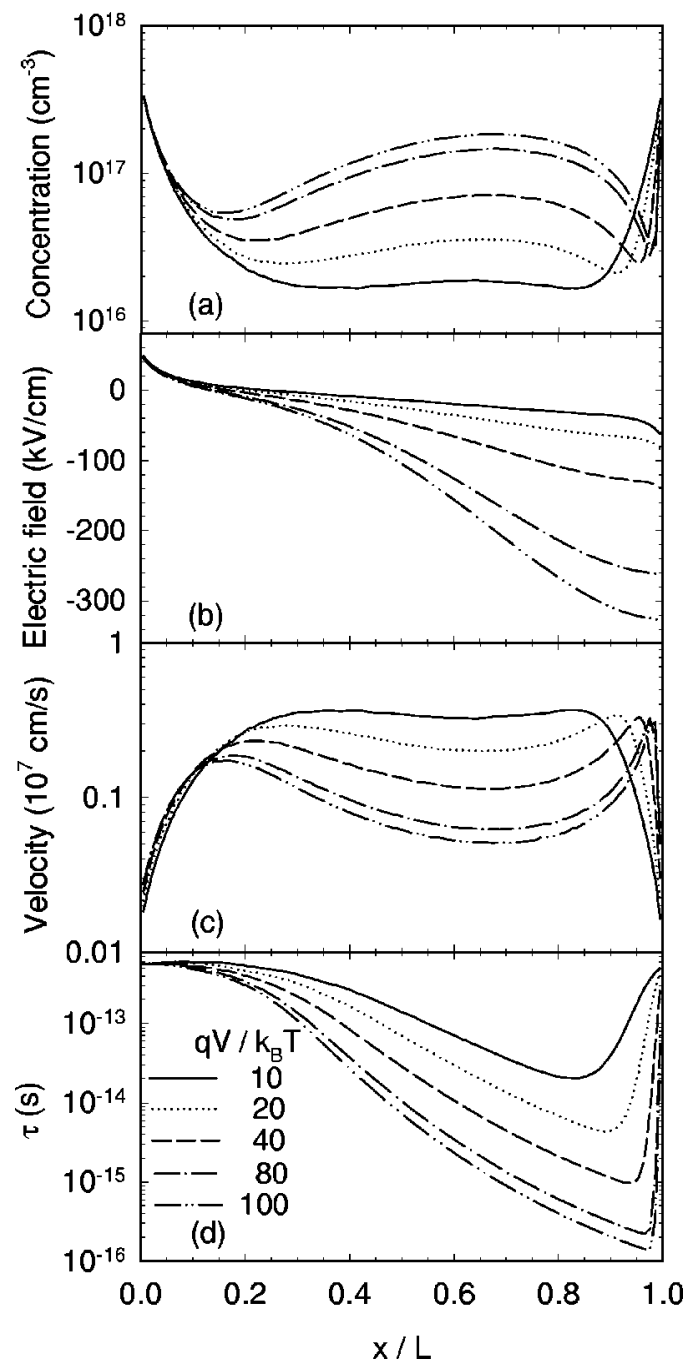

FIG. 4. Spatial profiles of (a) carrier concentration, (b) electric field, (c) average velocity, and (d) relaxation time along the active region of the sample as obtained by $\mathrm{MC}$ calculations for a $3 \mathrm{D}$ system, with $\alpha=-2$, and several values of the applied bias.

a function of bias for the simulated structure. For the sake of completeness, the inset in the same figure shows the corresponding Fano factor. The simulations prove that at the highest bias the sample indeed displays full shot noise, thus confirming theoretical expectations. We note that the values of the applied voltage needed to achieve full shot-noise conditions are much higher than those reported in Fig. 1. Focusing on the inset, we observe that the crossover between thermal and full shot noise does not follow the standard monotonic expression $\gamma=\operatorname{coth}\left(q V / 2 k_{B} T\right)$, but is mediated by a transition region that displays suppressed shot noise down to a minimum value. We remark that for the scattering parameters chosen here full shot noise appears at high voltages for which the $I-V$ characteristic is still far from the current saturation region. Therefore this full shot-noise regime cannot be related in any way to the presence of current saturation, as it happens in other systems like vacuum tubes ${ }^{19}$ or ballistic nondegenerate conductors. ${ }^{20-22}$ Rather, it constitutes an intrinsic property of the sample related to the diffusive transport regime of carriers (the same phenomenon can be ob-

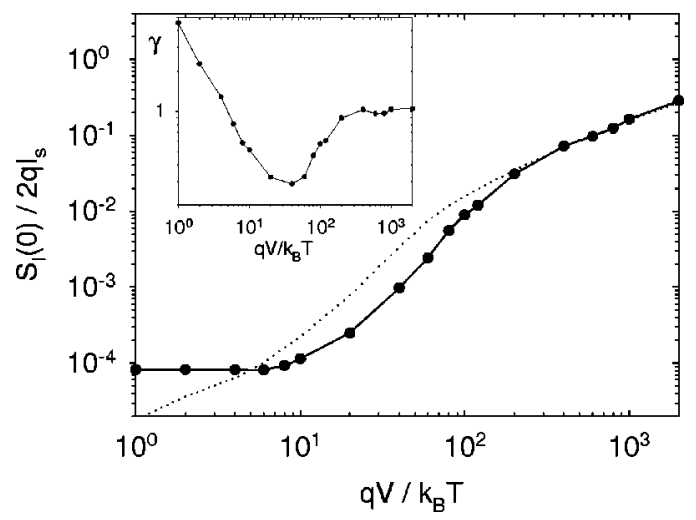

FIG. 5. Low-frequency current spectral density normalized to $2 q I_{S}$ as a function of the applied voltage for $\alpha=3 / 2$ (full circles, continuous line). The dotted line represents $2 q I$. Inset: Fano factor as a function of the applied voltage for the same case.

served in inelastic diffusive nondegenerate semiconductors, see below and Ref. 23). For the particular value of $\alpha=3 / 2$ the minimum value of the Fano factor in the anomalous region of the crossover is around 1/3. For other values of $\alpha$ $\in(-1.5,3 / 2)$ the value of the minimum is found to lay in the range $(1,1 / 3)$, as shown in Fig. 2. According to these results, we conclude that for sample lengths longer than the contact Debye screening length, $L>L_{D_{c}}$, and for $\alpha>-d / 2$, a nondegenerate elastic diffusive conductor exhibits the following three noise regimes: (i) thermal noise at low bias; (ii) suppressed shot noise, with a minimum value of the Fano factor determined by the energy dependence of the scattering time $(\alpha)$, at an intermediate bias range where the sample is under space-charge limited conditions and; (iii) full shot noise, independently of $\alpha$, at the highest bias range.

\section{B. Anomalous crossover between thermal and shot noise}

From the results of the previous section it emerges that the shot-noise suppression so found can be more generally interpreted in the context of an anomalous crossover between thermal and shot noise, as introduced in Ref. 23 for the case of macroscopic diffusive conductors where scattering is inelastic. To shed more light on this subject, we find of interest to carry out a comparison between the noise properties of an elastic nondegenerate conductor with those of an inelastic one. To this purpose we have performed simulations for a system with the same properties of that studied above but in the presence of inelastic collisions, using a scattering model already developed in Ref. 4. The results are reported in Fig. 6, which shows the low-frequency current spectral density for both elastic and inelastic diffusive conductors as a function of the current flowing through the samples. For the sake of completeness the inset reports the corresponding Fano factor. In both the elastic and inelastic cases, at increasing voltages we can distinguish three different noise regimes, corresponding to thermal, suppressed, and full shot-noise conditions. The degree of suppression of shot noise in the intermediate region is found to be much more pronounced in the inelastic case. This fact is more clearly illustrated in the inset of Fig. 6. Remarkably enough, the transition between 


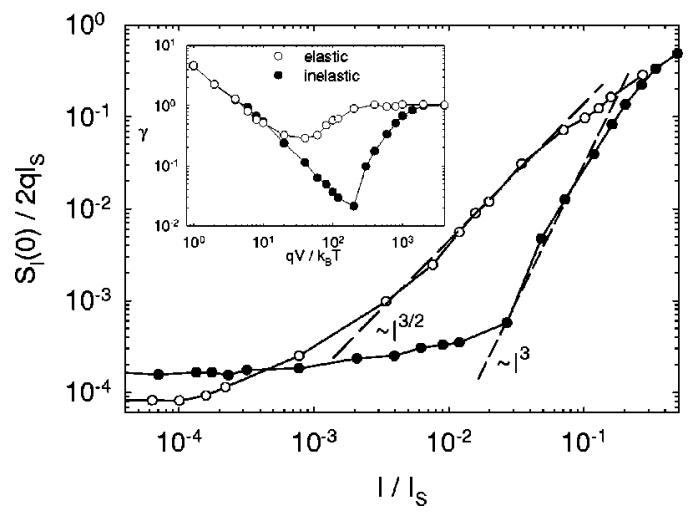

FIG. 6. Low-frequency current spectral density normalized to $2 q I_{S}$ as a function of the current normalized to its saturation value for $\alpha=3 / 2$ in the case of inelastic (full circles) and elastic (open circles) scattering. Symbols and solid lines refer to MC calculations, and dashed lines indicate power behaviors on the current in the transition (anomalous) region defining the crossover between thermal and shot noise. Inset: Fano factor as a function of the applied voltage for the same cases.

the thermal and the full shot-noise regimes in the inelastic case is mediated by a well defined anomalous crossover with a cubic dependence of the current spectral density upon the current, in agreement with the results of Ref. 23. For the elastic case, the crossover displays a power dependence with current to an exponent of three halves (see Fig. 6), which needs to be further investigated. We conclude that the anomalous crossover between thermal and full shot noise constitutes a unifying scenario to interpret shot-noise suppression in nondegenerate diffusive conductors.

\section{CONCLUSIONS}

We have presented a detailed analysis of the noise properties of nondegenerate elastic diffusive conductors. To this end, we have performed a complete set of Monte Carlo simulations on the basis of the indications provided by theoretical predictions. The simulations have confirmed the existence of enhanced shot noise when the exponent $\alpha$ of the energy dependence of the relaxation time satisfies the condition $\alpha$ $<-d / 2$, with $d$ the dimensionality of the system in momentum space. Furthermore, the presence of full shot noise is confirmed under different conditions, namely: (i) when the length of the sample is shorter than the contact Debye length, (ii) when $\alpha>-d / 2$ and the applied bias is high enough so that the transit time becomes the shortest time scale of the system, and (iii) when $\alpha=-d / 2$. The presence of enhanced shot noise for $\alpha<-d / 2$ is found to coincide with the analytical condition that the differential conductivity in energy space becomes negative. The presence of super-Poissonian values of the Fano factor is here interpreted as precursor of an electrical instability driven by a region of dynamical negative differential mobility inside the sample. This instability is associated with the bimodal spatial profile of the carrier average velocity, which is generated by the simultaneous presence of two distinct transport regimes: a ballistic one near to the contacts, and a diffusive one inside the structure. When the sample displays suppressed shot noise the Fano factor exhibits a minimum whose value depends on $\alpha$. This dependence is in reasonable agreement with analytical theories developed for space-charge limited conditions and $d=3$ in the range $-1.5<\alpha<0.5$. Outside this range analytical theories fail completely providing unphysical values. Since full shot noise is asymptotically reached at the highest bias, shot-noise suppression (and the corresponding minimum value of the Fano factor) is more generally interpreted in terms of an anomalous crossover between thermal and full shot noise. We believe that this investigation provides a comprehensive understanding of the noise properties of nondegenerate elastic diffusive conductors, and sheds interesting insight into the nonequilibrium noise properties of mesoscopic conductors.

\section{ACKNOWLEDGMENTS}

Partial support from the Ministerio de Ciencia y Tecnología and FEDER through the Ramon y Cajal Program and Project Nos. TIC2001-1754 and BFM2001-2159, from the Consejería de Educación y Cultura de la Junta de Castilla y León through Project No. SA057/02, and from the ItalySpain Joint Action of the MIUR Italy (Ref. IT109) and MCyT Spain (Ref. HI2000-0138) is gratefully acknowledged.
${ }^{1}$ For a recent review on shot noise in mesoscopic systems, see Ya. M. Blanter and M. Buttiker, Phys. Rep. 336, 1 (2000).

${ }^{2}$ C. W. Beenakker and M. Büttiker, Phys. Rev. B 46, 1889 (1992).

${ }^{3}$ K. E. Nagaev, Phys. Lett. A 169, 103 (1992).

${ }^{4}$ T. González, C. González, J. Mateos, D. Pardo, L. Reggiani, O. M. Bulashenko, and J. M. Rubí, Phys. Rev. Lett. 80, 2901 (1998).

${ }^{5}$ T. González, J. Mateos, D. Pardo, O. M. Bulashenko, and L. Reggiani, Phys. Rev. B 60, 2670 (1999).

${ }^{6}$ T. González, J. Mateos, D. Pardo, L. Reggiani, and O. M. Bulashenko, Physica B 272, 282 (1999).

${ }^{7}$ C. W. J. Beenakker, Phys. Rev. Lett. 82, 2761 (1999).

${ }^{8}$ H. Schomerus, E. G. Mishchenko, and C. W. Beenakker, Phys.
Rev. B 60, 5839 (1999).

${ }^{9}$ H. Schomerus, E. G. Mishchenko, and C. W. Beenakker, in Statistical and Dynamical Aspects of Mesoscopic Systems, edited by D. Reguera, G. Platero, L. L. Bonilla, and J. M. Rubí (Springer, Berlin, 2000), p. 96.

${ }^{10}$ K. E. Nagaev, Phys. Rev. Lett. 83, 1267 (1999).

${ }^{11}$ V. L. Gurevich and M. I. Muradov, J. Exp. Theor. Phys. 94, 1026 (2002).

${ }^{12}$ G. Gomila and L. Reggiani, Semicond. Sci. Technol. 15, 829 (2000).

${ }^{13}$ T. González, J. Mateos, D. Pardo, O. M. Bulashenko, and L. Reggiani, Semicond. Sci. Technol. 13, 714 (1998).

${ }^{14}$ K. Seeger, Semiconductor Physics, an Introduction (Springer, 
Berlin, 1985).

${ }^{15}$ L. Varani, L. Reggiani, T. Kuhn, T. González, and D. Pardo, IEEE Trans. Electron Devices ED-41, 1916 (1994).

${ }^{16}$ Sh. Kogan, Electronic Noise and Fluctuations in Solids (Cambridge University Press, Cambridge, England, 1996).

${ }^{17}$ From the simulations it is seen that there is charge injection from the contacts into the sample. This means that there should be a surface charge in the contacts to ensure the charge neutrality of the whole system. This charge is intrinsically accounted for by the self-consistent Poisson solver by assuming that the potential is constant at the contacts. The possible fluctuations of this charge on the current is neglected because this effect is expected to appear at high frequencies (comparable with those of the plasma), while we are interested in the low-frequency region of the spectrum. For a more detailed discussion of charge injection in solids, see M. A. Lampert and P. Mark, Current Injection in
Solids (Academic, New York, 1970). High-frequency effects have been analyzed in Y. Naveh, D. V. Averin, and K. K. Likharev, Phys. Rev. Lett. 79, 3482 (1997); K. Nagaev, Phys. Rev. B 57, 4628 (1998).

${ }^{18}$ M. P. Shaw, V. V. Mitin, E. Schöll, and H. L. Grubin, The Physics of Instabilities in Solid State Electron Devices (Plenum Press, New York, 1992), ISBN 0-306-43788-0.

${ }^{19}$ A. van der Ziel, Noise (Prentice-Hall, Englewood Cliffs, NJ, 1954).

${ }^{20}$ T. González, O. M. Bulashenko, J. Mateos, D. Pardo, and L. Reggiani, Phys. Rev. B 56, 6424 (1997).

${ }^{21}$ O. M. Bulashenko, J. Mateos, D. Pardo, T. González, L. Reggiani, and J. M. Rubí, Phys. Rev. B 57, 1366 (1998).

${ }^{22}$ O. M. Bulashenko, J. M. Rubí, and V. A. Kochelap, Phys. Rev. B 61, 5511 (2000).

${ }^{23}$ G. Gomila and L. Reggiani, Phys. Rev. B 62, 8068 (2000). 